\title{
Ectopic Pregnancy in Two Cats
}

\author{
Jia-San Zheng, Zheng Wang, Jia-Ren Zhang, Shuang Qiu, Ren-Yue Wei, Jing Nie \& Ting-Ting Zhu
}

\begin{abstract}
Background: Ectopic pregnancy mainly refers to tubal pregnancy and abdominal pregnancy. Tubal pregnancy presents as an implanted embryo that develops in the fallopian tubes, and is relatively common in humans. In animals, tubal pregnancy occurs primarily in primates, for example monkeys. The probability of a tubal pregnancy in non-primate animals is extremely low. Abdominal pregnancy is a type of ectopic pregnancy that occurs outside of the uterus, fallopian tube, ovary, and ligament (broad ligament, ovarian ligament, suspensory ligament). This paper describes two cases of ectopic pregnancy in cats. Cases: Cat 1. The presenting sign was a significant increase in abdominal circumference. The age and immune and sterilization status of the cat were unknown. On palpation, a $4 \mathrm{~cm}$, rough, oval-shaped, hard mass was found in the posterior abdomen. Radiographic examination showed three high-density images in the posterior abdomen. The fetus was significantly calcified and some feces was evident in the colon. The condition was preliminarily diagnosed as ectopic pregnancy. Cat 2. The owner of a 2-year-old British shorthair cat visited us because of a hard lump in the cat's abdomen. The cat had a normal diet and was drinking normally. Routine immunization and insect repulsion had been implemented. The cat had naturally delivered five healthy kittens two months previous. Radiographs showed an oval-shaped mass with a clear edge in the middle abdominal cavity. Other examinations were normal. The case was preliminarily diagnosed as ectopic pregnancy, and the pregnancy was surgically terminated. The ectopic pregnancies were surgically terminated. During surgery, the structures of the uterus and ovary of cat 1 were found to be intact and the organs were in a normal physiological position. Cat 1 was diagnosed with primary abdominal pregnancy. In cat 2, the uterus left side was small and the fallopian tube on the same side was both enlarged and longer than normal. Immature fetuses were found in the gestational sac. Thus, cat 2 was diagnosed with tubal ectopic pregnancy based on the presenting pathology.

Discussion: Cats with ectopic pregnancies generally show no obvious clinical symptoms. The ectopic fetus can remain within the body for several months or even years. Occasionally, necrotic ectopic tissues or mechanical stimulation of the ectopic fetus can lead to a systemic inflammatory response, loss of appetite, and apathy. The two cats in our report showed no significant clinical symptoms. To our knowledge, there have been no previous reports of the development of an ectopic fetus to maturity, within the abdominal cavity of felines, because the placenta of cats cannot support the growth and development of the fetus outside of the uterus. Secondary abdominal ectopic pregnancy, lacking any signs of uterine rupture is likely associated with the strong regenerative ability of uterine muscles. A damaged uterus or fallopian tube can quickly recover and rarely leaves scar tissue. In the present report, cat 1 showed no apparent scar tissue, nor signs of a ruptured ovary or fallopian tubes. It was diagnosed with primary ectopic abdominal pregnancy, which could arise from the descent of the fertilized egg from the fallopian tube into the abdominal cavity. There was an abnormal protrusion in left of the fallopian tubes in cat 2 , to which the gestational sac was directly connected. Based on pathological examination of the uterus, fallopian tubes, and gestational sac, the cat was diagnosed with a tubal pregnancy. Placental tissues and signs of fetal calcification were observed in both the fallopian tube and gestational sac.
\end{abstract}

Keywords: tubal pregnancy, abdominal pregnancy, feline, ectopic fetus, fallopian tube, gestational sac. 


\section{INTRODUCTION}

Ectopic pregnancy mainly refers to tubal pregnancy and abdominal pregnancy. Tubal pregnancy presents as an implanted embryo that develops in the fallopian tubes, and is relatively common in humans. In animals, tubal pregnancy occurs primarily in primates, for example monkeys [5]. The probability of a tubal pregnancy in non-primate animals is extremely low. The reasons for this include: the ectopically implanted embryo in the fallopian tubes has an insufficient nutrient and blood supply; maternal recognition of pregnancy is impaired, thereby leading to embryo death in early pregnancy [4]; and special mucus factors secreted by the fallopian tubes can inhibit attachment of the embryo to the tubes [9].Long-persisting abdominal fetuses frequently become calcified in women and are called lithopedions. In veterinary literature, long-lasting abdominal mineralized fetuses have been described in monkeys [4], cats [2], and rabbits [10].

Abdominal pregnancy is a type of ectopic pregnancy that occurs outside of the uterus, fallopian tube, ovary, and ligament (broad ligament, ovarian ligament, suspensory ligament. It can be divided into primary and secondary abdominal pregnancy, according to its origin. In primary abdominal pregnancy, the fertilized egg is implanted and grows within the abdominal cavity. Primary abdominal pregnancy usually occurs in primates, as they have a discoid villous placenta [4]. In secondary abdominal pregnancy, however, the uterus or ovary can be ruptured by external forces, mechanical stimulation, surgical trauma, and other factors, thereby causing the embryo or fertilized egg to enter the abdominal cavity, and attach to and develop in the omentum, mesentery, liver, kidney, spleen, or other organs [8,9]. Because of the reduced free and adequate nutrient and blood supply of the mesentery, embryos or fertilized eggs are often implanted in the mesentery.

\section{CASES}

Cat 1. The presenting sign was a significant increase in abdominal circumference. The age and immune and sterilization status of the cat were unknown. On palpation, a $4 \mathrm{~cm}$, rough, oval-shaped, hard mass was found in the posterior abdomen. Radiographic examination showed three high-density images in the posterior abdomen (Figure 1A). The fetus was significantly calcified and some feces was evident in the colon. No other examinations were performed because of economic considerations of the owner. The condition was preliminarily diagnosed as ectopic pregnancy. The pregnancy was surgically terminated after the owner's agreement was acquired. The skin and peritoneum were incised along the ventral median line to access the abdomen. A mummified fetus was wrapped within omental adipose tissues and had adhered to the abdominal wall (Figure 1B). The uterus and ovary were intact and no scar tissue was observed (Figure 1C). Other abdominal organs showed no significant changes. Fetal development was incomplete and the umbilical artery and vein were not found. The blood supply system was observed in the forearm artery and vein. Because of an insufficient blood supply, the fetus was mummified (Figure 1D).

Preoperative antibiotics (ceftriaxone $30 \mathrm{mg} / \mathrm{kg}$, i.v.) were administered. Preoperative and postoperative fasting lasted for $8 \mathrm{~h}$. Ceftriaxone (30 mg/kg, i.v.) was administered for 3 days after the surgery to prevent infection. The cat recovered well after surgery and appetite returned to normal. There were no other complications.

Cat 2. The owner of a 2-year-old British shorthair cat visited us because of a hard lump in the cat's abdomen. The cat had a normal diet and was drinking normally. Routine immunization and insect repulsion had been implemented. The cat had naturally delivered five healthy kittens two months previous. Radiographs showed an oval-shaped mass with a clear edge in the middle abdominal cavity (Figure 2A). Other examinations were normal. The case was preliminarily diagnosed as ectopic pregnancy, and the pregnancy was surgically terminated. During the surgery, a bulging, hard gestational sac was found in the fallopian tube on left side (Figure 2B and C). After the bulging area was longitudinally incised, three fetuses that were calcified and incompletely developed were found (Figure 2D). After surgery, tissue samples taken from the uterus' fallopian tube on the side containing the gestational sac were fixed in a $10 \%$ buffered formalin solution, paraffin embedded, sectioned, and stained with hematoxylin and eosin. The slides were examined and photographed using a light microscopeequipped with a video camera ${ }^{1}$.

Histopathological examination was performed on the uterus and fallopian tube on the side containing the gestational sac, as well as on the placenta and gestational sac of cat 2 . The structure of the uterus was clear, visible mucosal layer, muscle layer, serous layer, the 
mucosal layer was thickened, the interstitial tissue was loose, and interstitial cells were hypertrophied (Figure 3A). Typical decidualization changes were not observed, suggesting that the fertilized egg had not developed within the uterus. The uterine mucosa also showed hemosiderin deposition (Figure 3B). The gestational sac was found in the fallopian tube and its wall was calcified. Necrosis was observed in the placental tissue and calcification spots were observed (Figure 3C), suggesting that the fetuses had been dead for a relatively long time. The gestational sac showed calcified tubal muscles and serous layer (Figure 3D).

\section{DISCUSSION}

Cats with ectopic pregnancies generally show no obvious clinical symptoms. The ectopic fetus can remain within the body for several months or even years. Occasionally, necrotic ectopic tissues or mechanical stimulation of the ectopic fetus can lead to a systemic inflammatory response, loss of appetite, and apathy. The two cats in our report showed no significant clinical symptoms. Cases in which ectopic fetuses develop to maturity have been reported in humans; these cases often occur in the omentum, liver, and spleen [11]. To our knowledge, there have been no previous reports of the development of an ectopic fetus to maturity, within the abdominal cavity of felines, because the placenta of cats cannot support the growth and development of the fetus outside of the uterus [1].

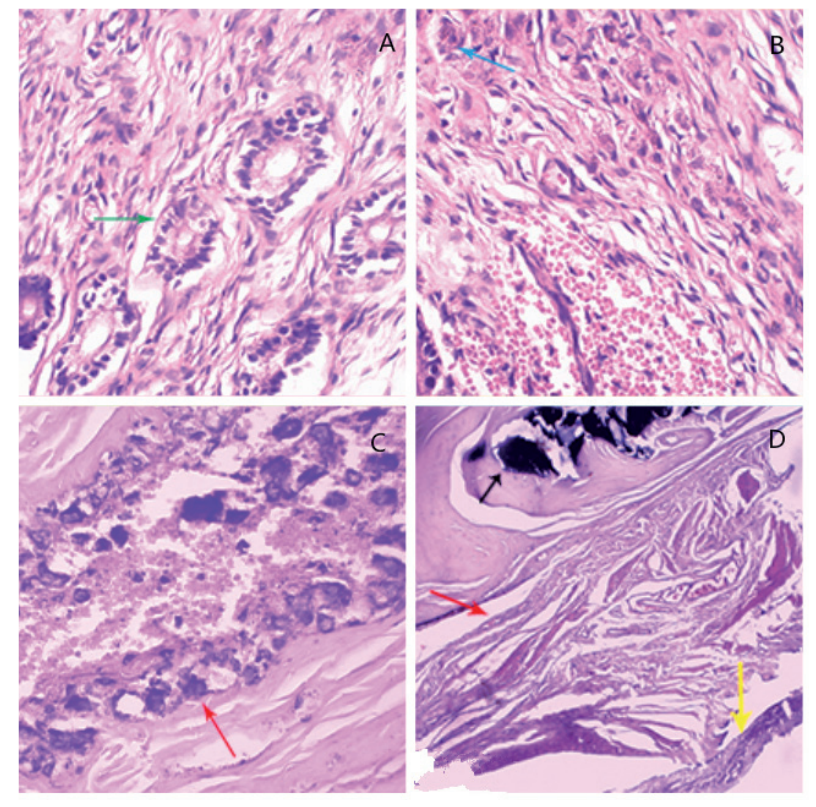

Figure 1. Cat 1: X-ray and surgical images. A- Radiograph shows three high-density masses in the abdomen. B- Gestational sac had adhered to omental adipose tissues. C- Uterus and ovary showed no injuries or scars. D- Calcified fetus with blood supply system located in the forearm artery and vein.
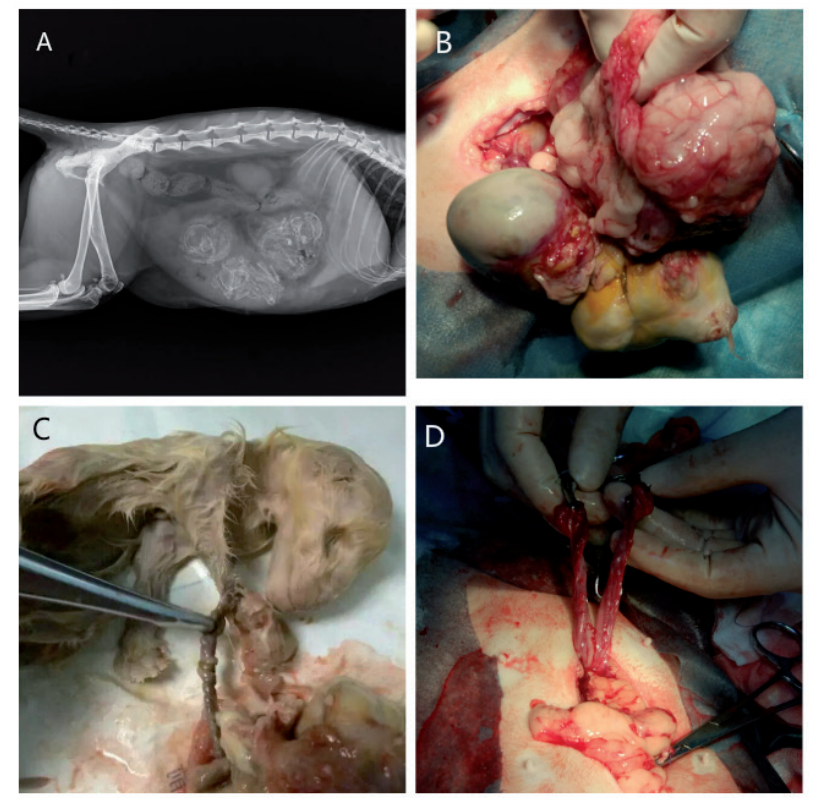

Figure 2. Cat 2: X-ray and surgical images. A- Radiographs show an oval mass in the abdominal cavity. B- Bulging gestational sac under the ovary on left side. C- Bulging gestational sac in the fallopian tube on left side. D- Fetuses in the gestational sac.
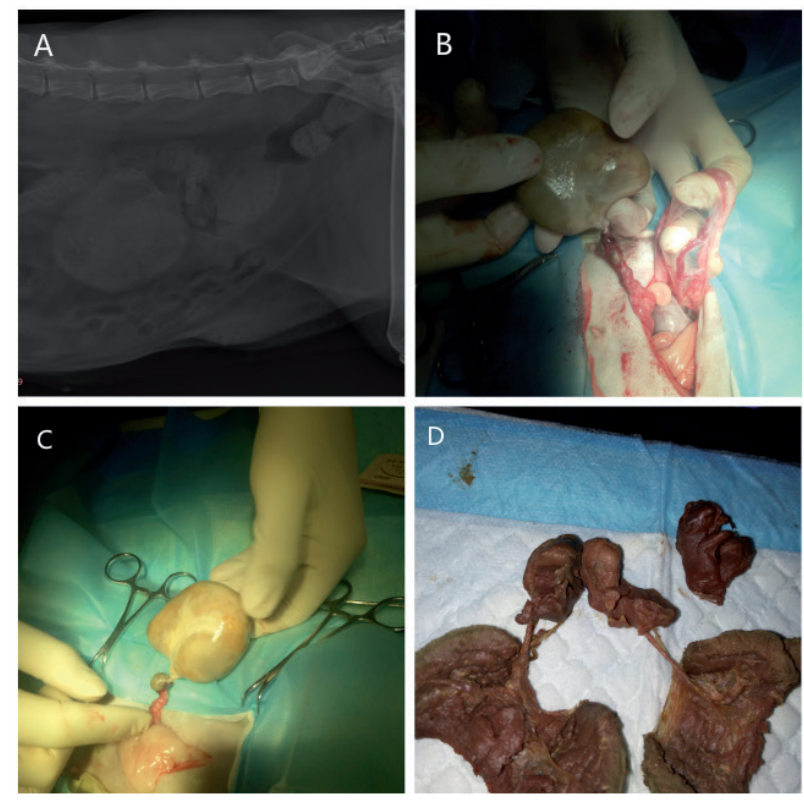

Figure 3. Cat 2: Uterine and tubal pathology pictures . A- Uterine tissue $(\times 40)$. The green arrow shows atypical decidualization changes, suggesting that the fertilized egg was not developed in the uterus. B- Uterine tissue $(\times 40)$. The blue arrow shows deposit of hemosiderin, suggesting a history of uterine bleeding. C- Section of the fallopian tube $(x 40)$. The red arrow shows the calcified gestational sac. D- Section of the gestational sac $(\times 40)$. The black arrow shows necrotic and calcified placental tissue, the red arrow shows the muscle layer of the fallopian tube, and the brown arrow shows the serous layer of the fallopian tube.

Compiling a list of differential diagnoses in some cases of primary and secondary ectopic pregnancies presents some challenges. Secondary abdominal ectopic pregnancy, lacking any signs of uterine rupture is likely associated with the strong regenerative ability of uterine muscles [7]. A damaged uterus or fallopian tube can quickly recover and rarely leaves scar tissue. 
One case report described a pregnant cat that was bitten by a dog, and subsequently sustained uterine rupture and an ectopic pregnancy; in that case, the formation of a uterine fistula and scar tissue was observed [6]. In the present report, cat 1 showed no apparent scar tissue, nor signs of a ruptured ovary or fallopian tubes. It was diagnosed with primary ectopic abdominal pregnancy, which could arise from the descent of the fertilized egg from the fallopian tube into the abdominal cavity [3]. Alternatively, severe abdominal pain caused by some diseases can lead to an increase in intrauterine pressure that could reverse the movement of the fertilized egg or fetus. The diagnosis of a primary abdominal ectopic pregnancy should be based on the following criteria: (1) no signs of estrus within the last three months, normal uterine and ovarian morphology, with no obvious scar tissue; (2) the ectopic fetus adheres only to abdominal organs and does not attach to the uterus or ovary. Cat 1 met the preceding diagnostic criteria. Cat 1 also had an abnormal blood supply system. The umbilical artery and vein were not found in the umbilicus, and the fetus was connected to the mother through an artery and vein in the forelimb. The reason for this finding might be due to the abnormal development of the yolk sac and allantois that was induced by unknown factors that led to the growth and development of the umbilical artery and vein in the forearm. It is also possible that the cat might have been exposed to teratogenic factors during pregnancy, like electromagnetic radiation or drugs, although a specific pathogenesis remains unclear.
There was an abnormal protrusion in left of the fallopian tubes in cat 2, to which the gestational sac was directly connected. Based on pathological examination of the uterus, fallopian tubes, and gestational sac, the cat was diagnosed with a tubal pregnancy. Placental tissues and signs of fetal calcification were observed in both the fallopian tube and gestational sac. Moreover, the gestational sac reflected the structure of the fallopian tube. The muscular and mucosal layers of the fallopian tube could be observed microscopically. However, the serous layer was not evident, and might have been absorbed or degraded during fetal calcification. One possible reason for tubal pregnancy is chronic inflammation that can cause transport dysfunction in the fallopian tubes. Impaired motility of tubal cilia, adhesions of the fallopian tube mucosa, as well as obstruction of the tubal cavity can all affect the movement of the fertilized egg to the uterus [7]. Cat 2 had given birth two months previous to examination, and had a history of inflammation, and we speculated that subsequent inflammation caused the blockage of one fallopian tube. The fertilized egg failed to descend into the uterus, and thereby resulted in a tubal pregnancy.

\section{MANUFACTURER \\ ${ }^{1}$ Nikon Corporation. Tokyo, Japan.}

Acknowledgments. This Work was supported by grants from National key research and development program of China (2016YFD0501008).

Declaration of interest. The authors declare no conflict of interests.

\section{REFERENCES}

1 Bodle T.J. 1979. Ectopic pregnancy in a cat. New Zealand Veterinary Journal. 27(12): 279-279.

2 Carrig C.B., Gourley I.M. \& Philbrick A.L. 1972. Primary abdominal pregnancy in a cat subsequent to ovariohysterectomy. Journal of the American Veterinary Medical Association. 160(3): 308-10.

3 Chavkin W. 1982. The rise in ectopic pregnancy-exploration of possible reasons. International Journal of Gynecology \& Obstetrics. 20(4): 341-350.

4 Corpa J.M. 2006. Ectopic pregnancy in animals and humans. Reproduction. 131(4): 631-640.

5 Jerome C.P. \& Hendrickx A.G. 1982. A tubal pregnancy in a rhesus monkey (Macaca mulatta). Veterinary Pathology. 19(3): 239-245.

6 Kumru I.H., Seyrek-Intas K., Tuna B., Celimli N. \& Seyrek-Intas D. 2007. Severe abdominal dog bite wounds in a pregnant cat. Journal of feline medicine and surgery. 9(6): 499-502.

7 Nack R.A. 2000. Theriogenology question of the month. Journal of the American Veterinary Medical Association. 217(2): 182-184.

8 Osenko A. \& Tarello W. 2014. A 7-Year-Old Extrauterine Pregnancy in a Cat. Case Reports in Veterinary Medicine. 2014: 1-3. Article ID 145064. 
9 Rosset E., Galet C. \& Buff S. 2011. A case report of an ectopic fetus in a cat. Journal offeline medicine and surgery. 13(8): 610-613.

10 Segura Gil P., Peris P.B., Martínez M.J., Porcel J.O. \& Arenas J.M.C. 2004. Abdominal pregnancies in farm rabbits. Theriogenology. 62(3): 642-651.

11 Tu J., Wang E. \& Shen J. 2016. Primary Hepatic Ectopic Pregnancy: A Case Report. The Journal of reproductive medicine. 61(3-4): 175-178. 